completion, project assessment remains necessary, resulted in education, removal, or change was completed by the project team. Evaluation of project was performed at 2 years post release.

Results During the initial 2-year project, 563 total requests were received with a closure rate of $97.9 \%$. Closed requests resulted in process change (25.9\%), removal $(0.6 \%)$, deemed necessary (40.7\%), education (32.78\%), and lack of response (2\%). Requests were categorized as relating to the EHR (70.9\%), pharmacy (2.3\%), documentation (4.9\%), compliance, $(1.8 \%)$, workflows $(6 \%)$, billing or coding $(2 \%)$, forms $(1.3 \%)$ and other (11.3\%).

Conclusions Programs to identify and evaluate processes that reduce the joy of healthcare can result in significant opportunities for change $(58 \%$ of requests in this project) via process change or elimination and education of team members on existing processes that are efficient.

\section{MEASURING PROFESSIONAL INTERPRETER USE AT THE IWK HEALTH CENTRE: A QUALITY IMPROVEMENT PROJECT}

Yasmeen Mansoor, Jeannette Comeau, Tania Wong. Dalhousie University and IWK Health Centre

\subsection{6/bmjoq-2021-IHI.26}

Background Patients with limited English proficiency (LEP) are subject to poorer health outcomes and improving interpreter access is a modifiable factor to improve health inequities faced by LEP patients.

Objectives To assess current interpreter provision for pediatric inpatients and their families at the IWK Health Centre by measuring the:

- proportion of non-English speaking patients identified at admission who received in-person interpretation during their hospital stay 
- proportion of patients who received in-person interpretation who were not identified as non-English speaking at the time of admission

- duration from the time of admission to the time of first interpreter encounter

Methods This was a cross-sectional study. All inpatient admissions at the IWK Health Centre from September 1, 2019 to March 1, 2021 were reviewed using admission records and the in-person interpreter database. The date, time, and duration of each admission to hospital and each interpreter encounter was used to calculate the outcome measures.

Results Of the 437 patients identified as non-English speaking on registration, $22.4 \%$ received in-person interpretation during their hospital stay, and this rate was $28.8 \%$ preCOVID-19 pandemic (pre-March 22, 2020), and 18.42\% post-COVID-19 pandemic (post-March 22, 2020), with a delta of $10.4 \%$ (95\% CI 2.2\%, 18.7\%) (figure 1). Of the 99 patients that received in-person interpretation, $20.8 \%$ of patients were inaccurately identified as English-speaking at time of registration. The mean time to the first in-person interpreter encounter was 38.2 hours after hospital admission $(\mathrm{SD}=59.1)$. Those who received in-person interpretation received a mean of 0.71 interpreter visits per hospital day $(\mathrm{SD}=0.53)$.

Conclusions This study offers baseline data illustrating that identification and provision of interpreter services for LEP is poor at the IWK. Based on these results, our centre will implement a universal screening question for interpreter preference at time of hospital admission as a quality improvement intervention to improve interpreter provision.

\section{USING QI METHODOLOGY TO INCREASE USAGE OF SEIZURE ACTION PLANS FOR PEDIATRIC PATIENTS WITH SEIZURES}

Dara VF Albert, Nancy Auer, James Herbst, William Parker, Anup D Patel. Nationwide Children's Hospital

\subsection{6/bmjog-2021-|HI.27}

Background Seizure Action Plans (SAP) have been proposed as an educational tool to help patients and caregivers be prepared for daily seizure care and emergencies. Meaningful clinical outcomes have been linked to the use of SAPs. Despite recognizing the importance of SAP use, 39\% of patients seen by epilepsy providers within our outpatient epilepsy center at Nationwide Children's Hospital received a SAP in 2016.

Objectives Increase and sustain the percentage of outpatient epilepsy center patients with seizure rescue medications who receive SAPs, from $39 \%$ to $85 \%$.

Methods A multi-disciplinary QI team developed key drivers and implemented interventions, such as an imbedded clinical pharmacist in the neurology clinic to assist with SAPs, clinic nursing staff providing SAP education at discharge, SAP enhancements to ease completion within the Electronic Medical Record (EMR), utilizing clinical decision support via the EMR to remind providers to complete SAPs, and provider and nursing education (figure 1). The team created a monthly report to monitor provider compliance for creating clinically indicated SAPs. The first half of 2016 was used as the preliminary baseline data.

\section{Seizure Action Plan Utilization in the Epilepsy Center \\ Interventions}

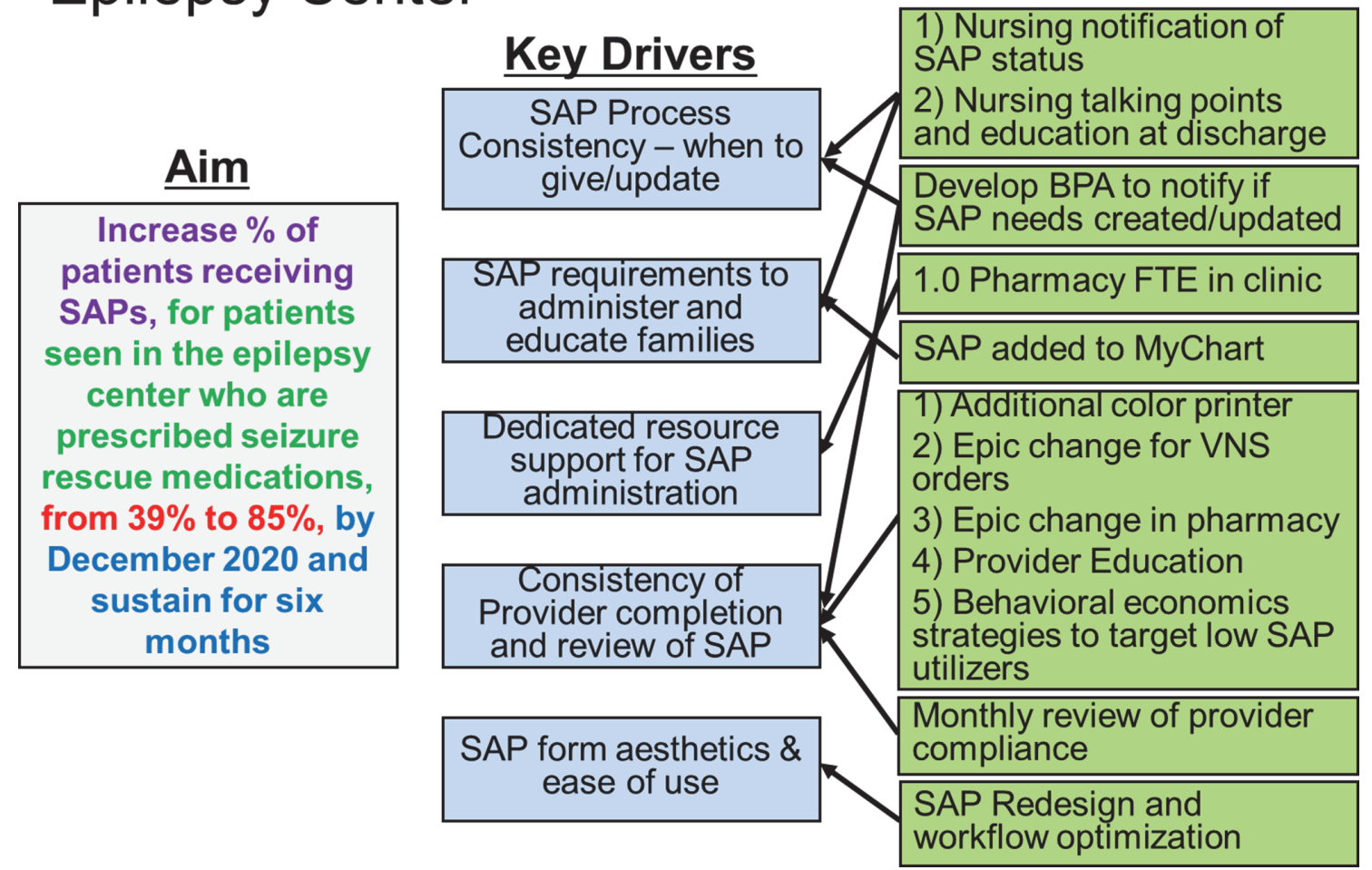

Abstract 27 Figure 1 This figure illustrates our key driver diagram including our specific project aim, important barriers identified (key drivers) and interventions put into place to overcome barriers 